\title{
Unveiling the environment-dependent mechanical properties of porous polypropylene separators
}

\author{
Shutian Yan ${ }^{1}$, Xinran Xiao ${ }^{1}$, Xiaosong Huang ${ }^{2}$, Xiaodong $\mathrm{Li}^{3}, \mathrm{Yue} \mathrm{Qi}^{4}$ ** \\ ${ }^{1}$ Department of Mechanical Engineering, Michigan State University, 2727 Alliance Dr, Lansing, \\ MI 48910, United States \\ ${ }^{2}$ Chemical Sciences \& Materials Systems Lab, General Motors Global Research \& Development, \\ 30500 Mound Rd, Warren, MI 48090, United States \\ ${ }^{3}$ Department of Mechanical and Aerospace Engineering, University of Virginia, Charlottesville, \\ Virginia 22904, United States \\ ${ }^{4}$ Department of Chemical Engineering and Materials Science, Michigan State University, East \\ Lansing, Michigan, 48824, United States
}

\begin{abstract}
Porous polypropylene (PP) is commonly used as separator materials for lithium ion batteries (LIB). Its mechanical properties, especially critical for abuse tolerance and durability of LIB, are subject to change in different environments. To capture the mechanical responses of a porous PP separator, its microstructure was mapped into separate atomistic models of bulk crystalline phases and oriented amorphous nanofibers. These structures were relaxed and stretched in vacuum, water, and dimethyl carbonate (DMC) using molecular dynamics (MD). The simulation results revealed DMC molecules penetrated into the amorphous PP nanofiber, and reduced the local density and the Young's modulus. In contrast, water increased the Young's modulus of the amorphous PP nanofiber. Furthermore, neither water nor DMC had any impact on the Young's modulus of the crystalline phase. These results suggest that the DMC induced separator softening was attributed to the strong attraction of the less-polar DMC solvent with the amorphous fibrous PP nanofibers.
\end{abstract}

Keywords: mechanical property; molecular dynamics; porous polypropylene

*Corresponding author: Yueqi@egr.msu.edu

(C) 2014. This manuscript version is made available under the Elsevier user license http://www.elsevier.com/open-access/userlicense/1.0/ 


\section{Introduction}

Porous polymers and their membranes have a wide range of applications, such as filtration [1], tissue scaffolds [2], and battery separators [3-5]. In all three cases, mechanical properties play an important role to their performance and function. For example: the filtration membranes should withstand the pressure of the flow; the scaffold should possess comparable mechanical properties to the tissue at the implantation site [2]; and the separator should maintain its structural integrity to defects (such as local intrusion of foreign particles introduced during battery assembly and the growth of dendrite) [5,6]. All of the above applications take place in a wet environment. Mechanical tests, however, are often conducted in air and a dry state using samples in a pre-wet condition, i.e. after being immersed in solutions for a certain time and dried in air before the mechanical testing. Therefore, the understanding of the impact of solution environment on the mechanical property of porous polymers is rather limited.

It has been observed that some mechanical property changes in solutions are recoverable, but others are not. For example, softening is observed in poly( $\varepsilon$-caprolactone) matrix composite scaffold [7] tested in water. A softening effect remained in oriented nanofiber polyurethane scaffold after pre-wetted in phosphate buffer [8]. In comparison, recoverable softening behaviors have been reported for a cellulose nanofiber reinforced polymer matrix composite in water [9] and a collagenous tissue in glycerol [10]. The former is attributed to the water layer formed between the hydrophilic fibers and hydrophobic polymer matrix. The water layer reduced the interaction between the fibers and the matrix. As this interaction is an important reinforcing mechanism in composites, the presence of water results in $\sim 80 \%$ reduction in modulus. Removing water, by drying the samples at $50^{\circ} \mathrm{C}$ for $48 \mathrm{~h}$, restores the interaction and hence the composite regains its mechanical property. For the case of collagenous tissues, the modulus reduction occurs in glycerol, when glycerol diffuses into the tissue, alters macromolecular interaction and destabilizes the collagen structures [11]. This softening is fully reversible when the sample is re-hydrated in normal saline [10]. Although dry perflurosulfonic acid (PFSA) polymer is not porous, water is necessary to form proton conductive pathways for fuel cells applications. It has also has been shown that the Young's modulus of hydrated PFSA decreases linearly with increasing water content [12], as water forms connected domains surrounded by the hydrophilic side chain of PFSA molecules. These examples illustrate that the environmentally 
sensitive mechanical properties of polymers are closely related to the atomic interaction of solution and the polymer.

The electrolyte solvent induced softening observed in a polypropylene (PP) separator is recoverable (unpublished experiment). A porous PP membrane is often used as the separator in Li-ion batteries to provide electrical insulation between the positive and negative electrodes, while allowing ionic transport through the pores. The mechanical properties of a PP separator have been measured in air, water, and several common electrolyte solvents, with or without a lithium salt by Sheidaei et al [13]. It is observed that, while water did not change the mechanical properties of the PP separators, the presence of electrolyte with or without lithium salt softened the PP separator by $\sim 50 \%$. This softening effect was not observed in the measurement with prewet samples [14]. In other words, the softening behavior of the PP separator occurred only when it was immersed in certain solutions and the solutions did not irreversibly alter the mechanical property of the PP separator. So far, the solution induced reversible softening behavior of the porous PP separator has not been satisfactorily explained.

We believe that the solution induced softening of the PP separator is due to its unique microstructure and its atomic interactions at molecular level, so that a multi-scale modeling approach is required. A typical view of the meso-scale structure of the Celgard 2400 porous PP separator is shown in Figure 1. The oriented elliptical pores and the parallel nanofibers with diameter 10 50nm are formed by a dry stretching process, which forces the stacked parallel lamellae to separate in the extrusion direction [15-17]. The stacked parallel lamellae patches are crystalline PP and the nanofibers are amorphous with polymer chains orientated along the fiber direction. The porosity is as high as $41 \%$ for Celgard 2400 [18]. Chou's [19] micromechanical model attempted to explain the softening phenomenon from the viewpoint of the surface energy. However, this model, along with surface energies obtained from wetting angle measurements and other macro-scale properties, can only predict a few percent of reduction in Young's modulus, an order of magnitude lower than the experimental result, suggesting that the underlining mechanism is still unclear.

When the separator is immersed in electrolyte solution, the nanofibers will be surrounded by the solution filling the pores. Due to the large surface to volume ratio, the Young's modulus of nanofibers will be more sensitive to the interactions with solution. According to Brochard et al 
[20], the surface effects must be taken into account to calculate the mechanical response for nanofibers with diameter smaller than $50 \mathrm{~nm}$. Recently, various atomic simulations have been used to calculate the impact of different chemical environments on the modulus of various nanostructures. Amer and Maguire [21] showed that when an isolated buckyball C60 molecule was immersed in a polar liquid like water and methanol, its bulk modulus increased while the C60 shrunk in volume. The predicted amount of shrinkage agreed with an early experiment by Wood et al. [22]. Density functional theory (DFT) calculation and experiments [23] have shown that the Young's modulus of a $\mathrm{ZnO}$ nanobelt increased in water due to increasing surface stress in the $\mathrm{ZnO}$ nanobelt. In investigations on biomaterials, it was found that the bending and twisting moduli of an $\alpha$-helix decreased slightly in water due to weak influence of water on the backbone hydrogen bond [24]. The Young's modulus of a collagen-like molecule decreased slightly due to a weak "lubricant" effect of water between stretched molecule and surrounding molecule $[25,26]$. These examples further illustrated that the mechanical properties of nanofibers tend to be highly environmentally sensitive.

The goal of this paper is to reveal the molecular origin of the softening behavior of a polymeric battery separator in electrolyte solvents via molecular dynamics (MD) simulations. More specifically, the Young's moduli of both crystalline and amorphous phases existing in the PP separator were computed with/without electrolyte solvent. This paper is organized as follows. Section II presents the method to build atomic structures in order to represent the different phases in battery separator in vacuum and in electrolyte solvent. Section III shows the MD details and statistics. Section IV compares the computed Young's moduli of both crystalline and amorphous phases in vacuum, DMC, water. The local density and order parameter of the polymer chain are analyzed to explain the origin of the softening behavior. Finally, Section V provides the conclusion.

\section{Atomic Model Construction}

As shown in the SEM images in Figure 1, the separator has an interconnected porous structure: nanometer sized pores surrounded by parallel nanofibers connecting stacked lamellae regions. Adam et al.'s scanning transmission electron microscope (STEM) dark field images [27] further illustrated that the lamellae regions had a unique crystalline structure, while most of the 
fibers are amorphous. In STEM, the fibers are either dark or tan in STEM, which is an indication of amorphous structure. We note that in principle it is necessary to simulate the connected crystalline and amorphous phases together to investigate the plastic deformation, during which the amorphous chain begins to distribute load to the crystalline phase, causing crystallographic slip [28,29]. However, the computing resources required in such simulations are prohibitive currently. For elastic deformation, crystalline and amorphous phases can be simulated separately and the predicted total elastic modulus was similar to experimental values for semicrystalline PP [28]. Thus, for the current work, the unique porous microstructure was mapped into separate atomic models of bulk crystalline and amorphous nanofibers. The atomic structures for each region are shown in Table 1 and the methods for building these atomic structures are detailed in this section.

\section{1 Pure crystalline (bulk and fiber in vacuum).}

Celgard 2400 is manufactured using isotatic PP (iPP) [14]. Two iPP phases exist, namely, $\alpha_{1}$ and $\alpha_{2}$, with $\alpha_{2}$ being more stable than $\alpha_{1}$ [29]. Therefore, the initial unit cell of crystalline PP was built based on the $X$ ray diffraction data on isotatic PP $\alpha_{2}$ phase [30,31]. The bulk crystalline was modeled with a $3 \mathrm{D}$ periodic structure. A $3 \times 1 \times 8$ supercell, containing 12 chains, was created as shown in Table 1. As seen from the side view, these chains were parallel to each other along chain direction ( $\mathrm{z}$ direction). The crystalline structure was relaxed at $298 \mathrm{~K}$ and at the experimental density with NPT dynamics for 180ps.

In addition, in order to compare with amorphous nanofibers, a crystalline nanofiber with a diameter of $3 \mathrm{~nm}$ or 12 chains, was also built by adding vacuum along both $\mathrm{x}$ and $\mathrm{y}$ directions in the simulation cell while maintaining $\mathrm{z}$ direction periodic (shown in Table 1). To simulate an isolated nanofiber, the vacuum layer thickness was larger than the non-bond cutoff distance (chosen as $9.5 \AA$ ). The crystalline nanofiber is then fully relaxed after $160 \mathrm{ps}$ NVT dynamics at $298 \mathrm{~K}$.

\section{2 Oriented amorphous nanofiber in vacuum}

As discussed in the introduction, the nanofibers of Celgard membrane are formed by stretching process. Stretching disentangles the PP chains, and increases the end-to-end distance of the chains, whose two ends are still tied to the adjoining crystalline lamellae. Additionally, 
stretching forces the chains in the nanofiber to preferentially align along the stretching direction [32]. Although the nanofibers may still have a little strain induced crystalline regions within the amorphous [27], only the amorphous region was modeled here as the representative structure.

In order to represent the atomic structure of the amorphous nanofiber, the average end-toend distance of the chains needed to be known because the Young's modulus of highly-oriented polymer depends on this parameter [34]. The end-to-end distance was estimated based on the processing condition. Experimentally, the stretching starts from the amorphous region of the Celgard precursor with a thickness of $6 \mathrm{~nm}$ [35]. After stretching, the fibers are formed with an average length of 100 150nm as estimated from the SEM image in Figure 1. Thus, the stretch ratio of the nanofibers was estimated as 20 times. To simulate a fully isotropic amorphous phase, an infinitely long PP chain was created in a 3D periodic simulation cell based on experimental density $0.86 \mathrm{~g} / \mathrm{cm}^{3}[36,37]$, more specifically, a chain with 648 monomers was tangled into a simulation cell of $3.75 \times 3.75 \times 3.75 \mathrm{~nm}^{3}$. After stretching 20 times, the average end-to-end distance of the chain became $75 \mathrm{~nm}$. If this chain were as rigid as it is in the crystalline phase, its end-to-end distance would have been $141 \mathrm{~nm}$. This means the average end-to-end distance of any two $-\mathrm{C}$ - atoms on the main chain in the orientated nanofiber region is folded to $53 \%$ compared with its end-to-end distance in the crystalline phase. Thus, this ratio is defined as the folding ratio.

Two oriented nanofibers with a 53\% folding ratio were built: one consisted of infinitely long chains (named as an oriented infinitely long chain nanofiber) and the other was made of shorter chains with end groups (named as an oriented finite chain nanofiber). The infinitely long chain closely mimicked bulk PP. In commercial commodity iPP, the molecular weight was at the level of $100,000 \mathrm{~g} / \mathrm{mol}$ for weight average $M_{\mathrm{w}}$ and 10,000 for number average $M_{\mathrm{n}}$ [38]. For comparison, the finite chain fiber was also simulated.

The basic steps to build infinitely long chain and finite chain nanofibers were the same. First, an initial amorphous nanofiber was created according to the folding ratio of 53\% oriented along the stretching direction ( $\mathrm{z}$ direction). Next, the nanofiber structure was continuously relaxed by several loading-unloading cycles. During loading and unloading, the potential energy was significantly reduced. The nanofiber was regarded as fully relaxed when the potential energy curve and the computed modulus were repeatable in loading and unloading. 


\subsubsection{Oriented infinitely long chain nanofiber}

A new method was developed to build amorphous nanofiber with oriented infinitely long chains. Since the crystalline PP structure had parallel infinitely long chains, it served as the starting point to build the amorphous nanofiber. A $2 \times 1 \times 27$ supercell containing 8 chains were created to build infinitely long chain nanofiber. To simulate the nanofiber, a vacuum layer thicker than the non-bond cutoff distance $(9.5 \AA)$ was added along both $\mathrm{x}$ and $\mathrm{y}$ directions while the chain direction ( $\mathrm{z}$ direction) was maintained as periodic.

Additional melting and compression steps were required to generate amorphous infinitely long chains with $53 \%$ folding ratio. A continuous compression with $1 \%$ strain decrement in the chain direction was applied at $500 \mathrm{~K}$, until the cell length along z direction reached $53 \%$ (as $93 \AA$ ) of the original length. At each compression strain, NVT dynamics was performed for 1ps, to allow folding and buckling of the chains. After the 53\% folding ratio was reached, the structure was then relaxed/melted at $700 \mathrm{~K}$ for $200 \mathrm{ps}$, and then linearly quenched to $298 \mathrm{~K}$ within $4 \mathrm{ps}$, followed by atomic relaxation for $1 \mathrm{~ns}$.

To further optimize the nanofiber structure, the relaxed fiber was subject to loadingunloading cycles. At each strain step, the fiber was relaxed for $150 \mathrm{ps}$. The method effectively reduced the potential energy from $160 \mathrm{cal} / \mathrm{mol}$ to $50 \mathrm{kcal} / \mathrm{mol}$ after $2 \sim 3$ loading-unloading cycles. However the Young's modulus did not converge until the $8^{\text {th }}$ unloading. In the final two loading-unloading cycles, the fiber was relaxed for 300ps at each strain step. The potential energy (variation was less than 1\%) and the stress-strain curve were nearly the same (with fitted Young's modulus variation was $\sim 0.2 \mathrm{GPa}$ ) in the final loading and unloading cycles.

\section{2. 2 Oriented finite chain nanofiber}

The initial structure to build oriented finite chain fiber was amorphous bulk with oriented chains. Initially, a straight chain with 80 monomers was built using Polymer Builder in Material

Studio. Then the two chains were packed in a periodic cell, with a packing density of $0.90 \mathrm{~g} / \mathrm{cm}^{3}$, using Amorphous Cell, a Monte Carlo sampling procedure to grow amorphous polymers according to atomic interactions $[39,40]$. An orientation bias along the chain direction ( $\mathrm{z}$ direction) was imposed during sampling. To keep the chains from being fully tangled or losing orientation, a low temperature of $150 \mathrm{~K}$ was used. The two chains in the as-built amorphous cell 
have orientation angles (between the end-to-end vector and $\mathrm{z}$ direction) of $15^{\circ}$ and $22^{\circ}$, and their end-to-end distance are $62.36 \AA$ and $54.56 \AA$, corresponding to folding ratio of $36 \%$ and $32 \%$.

Afterwards a $2 \times 2 \times 1$ supercell was created to include 8 oriented chains and the vacuum layer was added to form an isolated nanofiber. The loading-unloading cycles were repeated to optimize the nanofiber structure. After 2 3 loading-unloading cycles, the potential energy of the nanofiber fell significantly from the initial state of $-1750 \mathrm{kcal} / \mathrm{mol}$ to $-2050 \mathrm{kcal} / \mathrm{mol}$. The finite chain fiber was considered as fully relaxed after 3 cycles loading-unloading because the potential energy variation was less than $1 \%$ and the modulus variation was less than $0.01 \mathrm{GPa}$.

\subsection{Nanofibers in solvents}

Two solvents were investigated, namely water and DMC. DMC is a much less polar electrolyte solvent component used in Li ion batteries with low dielectric constant of 3.09 [41], compare to more polar solvent such as ethylene carbonate (EC) and water, with high dielectric constant of 89.1[42] and 78.39[43], respectively. Before submersing nanofiber into any solvent in simulations, the densities of the liquid solvents were computed. The computed density via NPT dynamics was $1.04 \mathrm{~g} / \mathrm{cm}^{3}$ for DMC and $0.97 \mathrm{~g} / \mathrm{cm}^{3}$ for water, which compares well with experimental density of $1.07 \mathrm{~g} / \mathrm{cm}^{3}$ for DMC [41] and $1.00 \mathrm{~g} / \mathrm{cm}^{3}$ for water at $298 \mathrm{~K}$. Additionally, stretching the pure liquid solvent structure led to zero Young's modulus, as expected.

The PP fiber-solvent mixtures were created by packing solvent molecules into the vacuum space around the isolated nanofiber. The number of molecules was determined by the vacuum space volume and the simulated bulk liquid density. The volume of the vacuum space was computed by deducting the nanofiber volume from the simulation cell volume, and the nanofiber volume was obtained by creating a solvent accessible surface to wrap it as shown in Figure 2a. Fine adjustments on the number of solvent molecules were made after structural relaxation to ensure that the nanofiber-solvent mixture was stress-free in $\mathrm{x}$ and $\mathrm{y}$ direction as shown in Figure 2b. The parameters for system size, including the cell size, the total number of atoms and the number of solvent molecules packed in each cell are summarized in Table 2.

\section{Molecular Dynamics Simulation details and statistics}

\subsection{Force Field}


The force field used here was COMPASS forcefiled [44] and the charges were default charges assigned by the force field. PP is a rather neutral system, so the atomic charges in PP were small. Occasionally, atomic charges were set zero to accelerate initial structural relaxations (the first several loading-unloading cycles to form the nanofibers), then followed by calculations with full atomic charge. The electrostatic energy summation method was Ewald [45], with an accuracy of $0.01 \mathrm{kcal} / \mathrm{mol}$ proved sufficient. Using higher Ewald accuracy like $0.00001 \mathrm{kcal} / \mathrm{mol}$ only led to $\sim 0.6 \mathrm{kcal} / \mathrm{mol}$ difference in total energy and less than $0.002 \mathrm{kcal} / \mathrm{mol} / \AA$ difference in force calculation. $\eta$, from which the summation range in real space and reciprocal space can be calculated, is automatically chosen by the program to balance the computational loads for real and reciprocal spaces. Using a more advanced particle mesh Ewald solver (PME) and different Ewald parameters (see supplementary material) did not change the main conclusion of this paper. In addition, the energy calculated by Ewald summation did not vary with the vacuum layer thickness, further confirming that PP was a relatively neutral system. On the other hand, the van

der Waals summation used an atom based cutoff method with a cutoff distance $9.5 \AA$. Comparing with the energy and stress recalculated using the same trajectories but with a much larger vdW cutoff distance of $30 \AA$, the energy and stress shifted by the same value at each strain, indicating the modulus was not dependent on the cutoff distance. The simulated solvent density (section 2.3) and PP bulk modulus (reported in section 4) all confirmed the accuracy of the force field. To further validate the results computed from COMPASS force field, comparison simulations with General AMBER force field [46] for PP and DMC and SPC/E for water [47] were also performed and the results are presented in the supplementary materials. Although the density values of water and DMC were more accurately predicted by AMBER and SPC/E, the relaxed structures of nanofiber in water and DMC remain the same trend.

\subsection{Simulation details and statistics}

All MD simulations were carried out with a time step of $1 \mathrm{fs}$ using a Nose-Hoover thermostat with a coupling constant of $10 \mathrm{fs}$ to maintain a constant temperature.

The method used to calculate the modulus was the constant strain method. The fiber was subject to deformation at a constant strain/cell-length increment in loading or decrement in unloading. The cell-length change at each strain step was $0.52 \AA$ for the crystalline fiber, $0.88 \AA$ for the infinitely long chain fiber, and $0.53 \AA$ for the finite chain fiber, along the $\mathrm{z}$ direction. The 
corresponding strain increment or decrement is $0.9 \%$ 1\%. At each strain step, the atomic structure was relaxed by NVT dynamics at $298 \mathrm{~K}$, which was higher than the glass transition temperature of PP (273K) [48]. The average potential energy and stress were then computed. The nanofiber stress was obtained by rescaling the stress of the simulation cell to the true volume of the nanofiber (the solvation accessible volume):

$$
\sigma_{\text {fiber }}=\sigma_{b o x} \times \frac{V_{b o x}}{V_{f i b e r}}
$$

The fiber true volume was approximated as the volume wrapped inside the accessible solvent surface with a small solvent radius $1.0 \AA$. Since fiber true volume at zero strain was used in equation (1), the stress calculated according to equation (1) was the engineering stress. The fiber engineering stress-strain curve was plotted and the Young's modulus was fitted within the linear elastic region. It should be noticed, as discussed in the previous section, that since the liquid solvent did not contribute to the total stress, the stress on the nanofiber in liquid could be obtained by the same rescaling method.

In order to obtain a strain-rate independent Young's modulus, fully relaxed structures were obtained at each strain level. The simulation time at each strain was determined separately for each system based on the energy and stress convergence criterion. The running average of variable $p$ at simulation step $n$ was defined as:

$$
\overline{p_{n}}=\frac{1}{n} \sum_{i=n_{0}}^{i=n} p_{n}
$$

where $n_{0}$ was the step starting to collect data, $n$ was the simulation step, and $p$ was a computed variable. In most cases, only the first $10 \%$ of data was excluded from the running average due to large fluctuation at the beginning. Taking step $I$ between step $n_{0}$ and $n$, an error value $e$ can be defined as the difference between the maximum and minimum values of running average between step $I$ and the final step $N$.

$$
\begin{gathered}
e=\overline{p_{\max }}-\overline{p_{\min }} \\
\overline{p_{\max }}=\max \left\{\overline{p_{I}}, \overline{p_{I+1}}, \cdots, \overline{p_{N}}\right\}
\end{gathered}
$$




$$
\overline{p_{\min }}=\min \left\{\overline{p_{I}}, \overline{p_{I+1}}, \cdots, \overline{p_{N}}\right\}
$$

$p$ could be considered as converged at step $I$, if the error/average ratio is less than $\pm 0.003 \%$. Using the same $I$ and $N$ range determined by satisfying the energy convergence criteria, the maximum error in stress was found be less than $0.008 \mathrm{GPa}$, which was considered to be well converged. To satisfy the potential energy and stress convergence criterion, the total simulation time and time started to converge at each strain were determined and summarized in Table 3.

The averaged Young's modulus was obtained by linear fitting of the averaged stressstrain values. The standard deviation of the modulus was calculated by using the maximum and minimum stress values at each strain in the fitting. Overall, the standard deviation of the Young's modulus was within $15 \%$ of the average.

As shown in Table 3, a slower convergence rate was observed in the infinitely long chain nanofiber, compared with the finite chain nanofiber, and the simulation time was extended accordingly. Furthermore, the simulation time for the PP-solvent mixture should be larger than 200ps because the single liquid bulk containing 150 molecules needed at least 200ps to reach equilibrium. Additionally, the effect of simulation time on the Young's modulus calculation was tested. For example, the relaxation time of the finite chain nanofiber at each strain was extended from 50ps to $160 \mathrm{ps}$, but Young's modulus was almost the same for the two relaxation times, further indicating the elastic Young's modulus simulated here was strain rate independent. This is consistent with Lee and Rutledge's simulation results [28].

\subsection{Polymer structural analysis}

To understand the variation of Young's moduli of PP in different phases and environments, the local atomic density was calculated as a function of the radial distance to the center of the fiber. First, the center of the fiber was calculated by taking the average $(X, Y)$ from all the atoms. Next, the fiber was divided into cylindrical shells with constant cross-section area $9 \pi \AA^{2}$. The atomic density inside each shell was calculated. With this method, the local density profile along the radius could be generated.

To further characterize the local orientation, an order parameter was defined [49,50]: 


$$
S=\frac{1}{2}\left\langle 3\left(\cos ^{2} \theta\right)-1\right\rangle
$$

where $<>$ is the structure average over all the chains in the nanofiber and $\theta$ is the angle between the $\mathrm{Z}$ direction and the vector that connects the two backbone carbon atoms separated by a fixed number of bonds. The number of bonds was chosen to be 6 , because one unit cell of iPP has 3 monomers with $6 \mathrm{C}-\mathrm{C}$ bonds. This meant in crystalline iPP, that the $\mathrm{C}$ atom on the main chain would rotate back to its original $X$ and $Y$ position after every $6 \mathrm{C}$ atoms. Therefore, $S$ is 1 for crystalline iPP structure and $S$ is 0 for a totally disordered amorphous structure.

\section{Results and discussions}

The simulated stress-strain curves for nanofibers in different environments were plotted in Figure 3, and the fitted Young's moduli were summarized in Table 4.

\subsection{Modulus of bulk crystalline iPP}

Table 4 listed the Young's modulus of bulk crystalline iPP phase along three lattice directions in comparison with experimental and previous MD results. The modulus along the chain direction $\left(\mathrm{E}_{\mathrm{c}}\right)$ is $49.5 \mathrm{GPa}$, which agrees well with experimental values $(36 \sim 42 \mathrm{GPa})$. For such an anisotropic crystalline structure, it is not surprising to see that the modulus along the directions perpendicular to the chain direction $\left(\mathrm{E}_{\mathrm{a}}\right.$ and $\left.\mathrm{E}_{\mathrm{b}}\right)$ is $\sim 3.6 \mathrm{GPa}$, one order of magnitude smaller than $E_{Z} . E_{X}$ and $E_{Y}$ in this calculation are smaller than values reported in previous MD simulations, suggesting the COMPASS force field [44] might slightly underestimate the nonbond interactions comparing to other force fields listed in Table 4. Overall, the COMPASS force field used in this simulation was able to predict the Young's modulus of PP with sufficient accuracy.

\subsection{Influence of orientation, molecular weight, and local density on nanofiber Young's modulus in vacuum}

Both the finite and infinitely long chain amorphous nanofibers had much smaller moduli than the crystalline nanofiber (290 660MPa vs. 43.4GPa). There was no direct measurement of the Young's modulus of the nanofiber for comparison. Nevertheless, our results are at the same order of that of polyethylene nanofiber, a similar thermoplastic polymer. The PE nanofiber of a 
diameter of $5 \mathrm{~nm}$ was reported to have a Young's modulus of $0.3 \mathrm{GPa}$ at 200K [60]. Further comparison indicated that the modulus of the finite chain fiber was smaller than that of the infinitely long chain fiber. This difference arises from chain orientations, molecular weight and local density variation in nanofibers.

The huge difference between crystalline and amorphous nanofibers indicates that the impact of chain orientations on the Young's modulus. The order parameter $\langle S\rangle$ was still around 1 for crystalline fiber. The order parameter at the end of simulation at zero strain was 0.26 for the amorphous infinitely long chain fiber, but was 0.00 for the amorphous finite chain fiber. In fact, the folding ratio reduced to $28 \%$ for the finite chain fiber while it was maintained at $52 \%$ for the infinitely long chain. This trend is consistent with that highly ordered polymer structures having higher moduli. This trend also agrees well with our density simulations for amorphous PP bulk at different molecular weights (in the supplementary material) and the results of coarse grain simulations of PE thin film, where the bulk density of films increased with the molecular weight and the computed density from the molecules with large molecular weight agreed better with experimental data [61].

As a result of the differences in orientation and molecular weight of these fibers, the local density profile of the nanofibers in vacuum varies. The density profiles for the crystalline fiber, the infinitely long chain fiber and the finite chain fiber at zero strain are shown in Figure 4a 4c with square solid symbols. Note that the density is not uniform in nanofibers. Each nanofiber could be divided into two regions: the core region where the density is almost constant and the surface layer where the density gradually decreases to 0 . The average density in the core region was also computed and shown by a straight line. The finite chain nanofiber has a lower average core density $\left(0.83 \mathrm{~g} / \mathrm{cm}^{3}\right)$ than that of the infinitely long chain $\left(0.84 \mathrm{~g} / \mathrm{cm}^{3}\right)$ as shown in Figure $4 \mathrm{~b}$

and $4 \mathrm{c}$. Both are much lower than the core density of crystalline nanofiber $\left(0.93 \mathrm{~g} / \mathrm{cm}^{3}\right)$. Higher densities lead to higher Young's moduli. The chains in the surface layer are more diffused and loosely packed, resulting in lower density, suggesting that as the nanofiber diameter gets smaller, the larger surface to volume ratio can further soften the fiber, and a so-called "size-effect" is expected [60].

\subsection{Comparisons of softening behavior among different fiber structures and solvents}


By comparing the crystalline fiber modulus obtained in vacuum and in DMC in Figure $3 \mathrm{a}$, it was found that the Young's modulus increased about 7\% along the chain direction. In contrast, the Young's modulus of the infinitely long chain fiber in Figure $3 \mathrm{~b}$ reduced from 0.66 GPa in vacuum to $0.07 \mathrm{GPa}$ in DMC, an almost $89 \%$ modulus reduction. The Young's modulus of the finite chain fiber in Figure $3 \mathrm{c}$ reduced from $0.29 \mathrm{GPa}$ in vacuum to $0.01 \mathrm{GPa}$ in $\mathrm{DMC}$, a 97\% modulus reduction. On the other hand, the Young's modulus of the finite chain fiber in Figure $3 \mathrm{c}$ increased from $0.29 \mathrm{GPa}$ in vacuum to $0.59 \mathrm{GPa}$ in water, almost $100 \%$ stiffening.

The softening behavior of the amorphous PP fiber in DMC was caused by penetration of DMC into the amorphous fiber. A comparison of the relaxed structure in vacuum, in DMC and in water at zero strain is summarized in Table 5. DMC could penetrate into amorphous PP nanofibers and even dissolve the finite chain nanofiber. The penetrated DMC molecules reduced the local density of the amorphous fiber as shown in Figure 4 and thus reduced the Young's modulus of the amorphous PP nanofiber. Particularly, the core density decreased from $0.83 \mathrm{~g} / \mathrm{cm}^{3}$ to $0.51 \mathrm{~g} / \mathrm{cm}^{3}$ for the finite chain fiber in DMC, and from $0.84 \mathrm{~g} / \mathrm{cm}^{3}$ to $0.75 \mathrm{~g} / \mathrm{cm}^{3}$ for the infinitely long chain fiber. To explain the softening behavior, Chou et al [19] had to assume that the fibrous area in the PP separator splits into narrower strips upon loading in solvent, and our simulations also support this hypothesis. Meanwhile the surface layer became more diffused. The surface layer thickness increased from $12.7 \AA$ to $32.9 \AA$ for the finite chain fiber, and from $12.0 \AA$ to $17.6 \AA$ for the infinitely long chain fiber, respectively. This is consistent with the greater reduction of the Young's modulus observed in finite chain fiber when being immersed in DMC. This is also similar to the significant softening observed in hydrated collagen microfibrils owing to looser molecular packing [62]. It needs to be noted that the softening effect is also related to the large surface to volume ratio in nanofibers. It is expected that as the simulated fiber diameter increases, the Young's modulus will also increase [63]. The nanofiber diameter in our simulations was $\sim 5 \mathrm{~nm}$, while in Celgard 2400 their size are around $\sim 10 \sim 50 \mathrm{~nm}$ in diameter. Therefore, the amount of reduction of the Young's modulus in DMC for nanofibers in commercial separators will be smaller than that has been calculated here.

On the other hand, it was observed that DMC could not penetrate into the crystalline fiber. The core density of crystalline fiber increased from $0.93 \mathrm{~g} / \mathrm{cm}^{3}$ in vacuum to $0.96 \mathrm{~g} / \mathrm{cm}^{3}$ in DMC due to the pressure imposed by solvent. This explained the slight increase of Young's 
modulus in crystalline nanofiber in DMC. To rule out the molecular weight effect, a finite chain crystalline fiber was also built, relaxed, and submersed in DMC. No DMC penetration was observed. These results indicates that DMC could not dissolve the crystalline structure in the separator, no matter whether the crystalline phase was in its bulk form or nanofiber form. This observation suggested that although DMC dissolved the amorphous finite chain fiber in in our simulation, DMC should not dissolve the whole separator, since the ends of the amorphous nanofibers are tied to the crystalline regions. In fact, soaking 41.5mg Celgard 2400 separator in $20 \mathrm{~mL}$ DMC for 1 week did not show any sign of dissolution (unpublished experiment). Since the crystalline phase was not softened by DMC, we can understand why PP softening occurs only immediately after it is stretched passing the yield point, characterized by the formation of fibers and pores [64], but not before pore formation. This was because the initial structure of PP is highly crystallized [64], which could not be softened as we just revealed in the simulation.

The main reason why DMC penetrate into the amorphous PP fiber but not the crystalline PP fiber was due to the interchain interaction difference. To quantify the interchain interaction, we computed the cohesive energy density. The value is $1.03 \times 10^{8} \mathrm{~J} / \mathrm{m}^{3}$ and $1.48 \times 10^{8} \mathrm{~J} / \mathrm{m}^{3}$ for amorphous infinitely long chain fiber and crystalline fiber, respectively. These results were consistent with stronger interchain interaction and higher density in crystalline fiber.

In contrast to DMC, water stiffened the finite chain nanofiber. Not only was no water penetration into nanofiber observed, the core density of the finite chain nanofiber also increased from $0.83 \mathrm{~g} / \mathrm{cm}^{3}$ to $0.86 \mathrm{~g} / \mathrm{cm}^{3}$. Consequently, the Young's modulus of the finite chain nanofiber increased in water as shown in Figure 3c. Although only the finite chain fiber was simulated, this finding should be also applicable to infinitely long chain fibers, due to the very hydrophobic nature of PP. Furthermore, one could expect that ethylene carbonate (EC), another main electrolyte solvent component for Li ion batteries, has nearly the same effect on the mechanical properties of PP separator as water. EC is also even more polar than water, with even higher dielectric constant [42] than water. Interestingly, it has been observed that the amount of softening of separator in EC/DMC solvent is indeed lower than that in DMC solvent [13]. Further studies on mixed electrolyte solvents with $\mathrm{LiPF}_{6}$ are required to understand and predict the separator mechanical behavior in lithium ion batteries. The softening effect of the separator is important in the overall battery cell modulus used in battery pack design and crash simulations. 
As the softest component in a typical battery cell, the mechanical behavior of the separator determines the overall cell modulus at small strains [65].

\section{Conclusion}

In summary, detailed MD simulations were performed to investigate the mechanical responses of a porous PP separator in vacuum, in DMC, and in water. It was found that the crystalline region retained its Young's modulus in DMC. However, the amorphous nanofibers were softened by an order of magnitude in DMC. This was due to the strong attraction of the DMC solvent to the amorphous PP nanofibers and their high surface to volume ratio. DMC could penetrate into the amorphous nanofiber, which resulted in a Young's modulus reduction to one tenth of its original value. In contrast, a polar solvent, such as water, could increase the Young's modulus by slightly squeezing the amorphous fiber due to the repulsive interaction. Since the separator/solvent interaction changes with time and temperature, additional studies are required to fully illustrate the impact of solvents on separator mechanical properties. Furthermore, a micro-mechanics model that taking the input from atomic simulations is needed to predict the overall macro scale Young's modulus measured in experiments.

\section{Acknowledgments}

We acknowledge Pengfei Li's assistant in performing comparative simulations with AMBER. Qi is grateful for the support from National Science Foundation under Grant No. CMMI-1235092. Xiao acknowledges the support from National Science Foundation under Grant No. CMMI-1030821. 


\section{Reference}

1. Barhate, R. S., and Seeram Ramakrishna. 2007. "Nanofibrous Filtering Media: Filtration Problems and Solutions from Tiny Materials." Journal of Membrane Science 296(1-2): 1-8. doi:10.1016/j.memsci.2007.03.038.

2. Hutmacher, Dietmar W. 2000. "Scaffolds in Tissue Engineering Bone and Cartilage." Biomaterials, Orthopaedic Polymeric Biomaterials: Applications of Biodegradables 21(24): 2529-43. doi:10.1016/S0142-9612(00)00121-6.

3. Arora, Pankaj, and Zhengming (John) Zhang. 2004. "Battery Separators." Chemical Reviews 104(10): 4419-62. doi:10.1021/cr020738u.

4. Zhang, Sheng Shui. 2007. "A Review on the Separators of Liquid Electrolyte Li-Ion Batteries.” Journal of Power Sources 164(1): 351-64. doi:10.1016/j.jpowsour.2006.10.065.

5. Huang, Xiaosong. 2011. "Separator Technologies for Lithium-Ion Batteries." Journal of Solid State Electrochemistry 15(4): 649-62. doi:10.1007/s10008-010-1264-9.

6. Santhanagopalan, Shriram, Premanand Ramadass, and John (Zhengming) Zhang. 2009. "Analysis of Internal Short-Circuit in a Lithium Ion Cell." Journal of Power Sources, XIth Polish Conference on Fast Ionic Conductors 2008, 194(1): 550-57. doi:10.1016/j.jpowsour.2009.05.002.

7. Leung, Linus H., and Hani E. Naguib. 2012. "Characterization of the Viscoelastic Properties of Poly( $\varepsilon$-Caprolactone)-hydroxyapatite Microcomposite and Nanocomposite Scaffolds." Polymer Engineering \& Science 52(8): 1649-60. doi:10.1002/pen.23108.

8. Yeganegi, Masoud, Rita A. Kandel, and J. Paul Santerre. 2010. "Characterization of a Biodegradable Electrospun Polyurethane Nanofiber Scaffold: Mechanical Properties and Cytotoxicity." Acta Biomaterialia 6(10): 3847-55. doi:10.1016/j.actbio.2010.05.003.

9. Dagnon, Koffi L., Kadhiravan Shanmuganathan, Christoph Weder, and Stuart J. Rowan. 2012. "Water-Triggered Modulus Changes of Cellulose Nanofiber Nanocomposites with Hydrophobic Polymer Matrices." Macromolecules 45(11): 4707-15. doi:10.1021/ma300463y.

10. Wells, P.B., A.T. Yeh, and J.D. Humphrey. 2006. "Influence of Glycerol on the Mechanical Reversibility and Thermal Damage Susceptibility of Collagenous Tissues." IEEE Transactions on Biomedical Engineering 53(4): 747-53. doi:10.1109/TBME.2006.870232.

11. Yeh, Alvin T., Bernard Choi, J. Stuart Nelson, and Bruce J. Tromberg. 2003. "Reversible Dissociation of Collagen in Tissues." Journal of Investigative Dermatology 121(6): 1332-35. doi:10.1046/j.1523-1747.2003.12634.x.

12. Qi, Yue, and Yeh-Hung Lai. 2011. "Mesoscale Modeling of the Influence of Morphology on the Mechanical Properties of Proton Exchange Membranes." Polymer 52(1): 201-10. doi:10.1016/j.polymer.2010.11.013.

13. Sheidaei, Azadeh, Xinran Xiao, Xiaosong Huang, and Jonathon Hitt. 2011. "Mechanical Behavior of a Battery Separator in Electrolyte Solutions." Journal of Power Sources 196(20): 8728-34. doi:10.1016/j.jpowsour.2011.06.026. 
14. Love, Corey T. 2011. "Thermomechanical Analysis and Durability of Commercial MicroPorous Polymer Li-Ion Battery Separators." Journal of Power Sources 196(5): 2905-12. doi:10.1016/j.jpowsour.2010.10.083.

15. Chen, R. T., C. K. Saw, M. G. Jamieson, T. R. Aversa, and R. W. Callahan. 1994. "Structural Characterization of Celgard ${ }^{\circledR}$ Microporous Membrane Precursors: Melt-Extruded Polyethylene Films." Journal of Applied Polymer Science 53(5): 471-83. doi:10.1002/app.1994.070530502.

16. Lee, Sang-Young, Soon-Yong Park, and Heon-Sik Song. 2006. "Lamellar Crystalline Structure of Hard Elastic HDPE Films and Its Influence on Microporous Membrane Formation.” Polymer 47(10): 3540-47. doi:10.1016/j.polymer.2006.03.070.

17. Sarada, T., L. C. Sawyer, and M. I. Ostler. 1983. "Three Dimensional Structure of Celgard® Microporous Membranes." Journal of Membrane Science 15(1): 97-113. doi:10.1016/S0376-7388(00)81364-2.

18. http://www.celgard.com/pdf/library/Celgard_Product_Comparison_10002.pdf

19. Chou, C. J., A. Hiltner, and E. Baer. 1986. "The Role of Surface Stresses in the Deformation of Hard Elastic Polypropylene." Polymer 27(3): 369-76. doi:10.1016/0032-3861(86)901527.

20. Brochard, L., M. Vandamme, and R. J. -M. Pellenq. 2012. "Poromechanics of Microporous Media." Journal of the Mechanics and Physics of Solids 60(4): 606-22. doi:10.1016/j.jmps.2012.01.001.

21. Amer, Maher S., and John F. Maguire. 2009. "On the Compressibility of C60 Individual Molecules." Chemical Physics Letters 476(4-6): 232-35. doi:10.1016/j.cplett.2009.06.010.

22. Wood, Jonathan R., Mark D. Frogley, Erwin R. Meurs, Andrew D. Prins, Ton Peijs, David J. Dunstan, and H. Daniel Wagner. 1999. "Mechanical Response of Carbon Nanotubes under Molecular and Macroscopic Pressures." The Journal of Physical Chemistry B 103(47): 10388-92. doi:10.1021/jp992136t.

23. Yang, Yingchao, Guofeng Wang, and Xiaodong Li. 2011. "Water Molecule-Induced Stiffening in ZnO Nanobelts." Nano Letters 11(7): 2845-48. doi:10.1021/nl201237x.

24. Choe, Seungho, and Sean X. Sun. 2005. "The Elasticity of A-Helices." The Journal of Chemical Physics 122(24): 244912. doi:10.1063/1.1940048.

25. Zhang, Dajun, Uday Chippada, and Kenneth Jordan. 2007. "Effect of the Structural Water on the Mechanical Properties of Collagen-like Microfibrils: A Molecular Dynamics Study." Annals of Biomedical Engineering 35(7): 1216-30. doi:10.1007/s10439-007-9296-8.

26. Gautieri, Alfonso, Monica I. Pate, Simone Vesentini, Alberto Redaelli, and Markus J. Buehler. 2012. "Hydration and Distance Dependence of Intermolecular Shearing between Collagen Molecules in a Model Microfibril." Journal of Biomechanics 45(12): 2079-83. doi:10.1016/j.jbiomech.2012.05.047.

27. Adams, W. Wade, D. Yang, and Edwin L. Thomas. 1986. "Direct Visualization of Microstructural Deformation Processes in Polyethylene." Journal of Materials Science 21 (7): 2239-53. doi:10.1007/BF01114262. 
28. Lee, Sanghun, and Gregory C. Rutledge. 2011. "Plastic Deformation of Semicrystalline Polyethylene by Molecular Simulation." Macromolecules 44(8): 3096-3108. doi:10.1021/ma1026115.

29. Romanos, Nikolaos A., and Doros N. Theodorou. 2010. "Crystallization and Melting Simulations of Oligomeric al Isotactic Polypropylene." Macromolecules 43(12): 5455-69. doi:10.1021/ma100677f.

30. Jones, A. Turner, Jean M. Aizlewood, and D. R. Beckett. 1964. "Crystalline Forms of Isotactic Polypropylene." Die Makromolekulare Chemie 75(1): 134-58. doi:10.1002/macp.1964.020750113.

31. Immirzi, A., and P. Iannelli. 1988. "Whole-Pattern Approach to Structure Refinement Problems of Fibrous Materials: Application to Isotactic Polypropylene." Macromolecules 21(3): 768-73. doi:10.1021/ma00181a036.

32. Ward, I. M., and J. Sweeney. 2004. An Introduction to The Mechanical Properties of Solid Polymers. John Wiley \& Sons Ltd, West Sussex, England.

33. Paavilainen, S., J. L. McWhirter, T. Róg, J. Järvinen, I. Vattulainen, and J. A. Ketoja. 2012. "Mechanical properties of cellulose nanofibrils determined through atomistic molecular dynamics simulation." Nordic Pulp and Paper Research Journal 27(2): 282-86.

34. Gedde, Ulf. W. 1999. Polymer physics. Kluwer Academic,The Netherlands.

35. Hild, S., W. Gutmannsbauer, R. Lüthi, J. Fuhrmann, and H.-J. Güntherodt. 1996. "A Nanoscopic View of Structure and Deformation of Hard Elastic Polypropylene with Scanning Force Microscopy.” Journal of Polymer Science Part B: Polymer Physics 34(12): 1953-59. doi:10.1002/(SICI) 1099-0488(19960915)34:12<1953::AID-POLB1>3.0.CO;2-R.

36. Liu, Hongyi, Yan Li, Wendy E. Krause, Orlando J. Rojas, and Melissa A. Pasquinelli. 2012. "The Soft-Confined Method for Creating Molecular Models of Amorphous Polymer Surfaces." The Journal of Physical Chemistry B 116(5): 1570-78. doi:10.1021/jp209024r.

37. Cozmuta, Ioana, Mario Blanco, and William A. Goddard. 2007. "Gas Sorption and Barrier Properties of Polymeric Membranes from Molecular Dynamics and Monte Carlo Simulations." The Journal of Physical Chemistry B 111(12): 3151-66. doi:10.1021/jp062942h.

38. Sadeghi, Farhad, Abdellah Ajji, Pierre J. Carreau. 2007. "Analysis of microporous membranes obtained from polypropylene films by stretching." Journal of Membrane Science 292(1-2): 62-71. doi:10.1016/j.memsci.2007.01.023.

39. Flory, P. J. 1974. "Foundations of Rotational Isomeric State Theory and General Methods for Generating Configurational Averages." Macromolecules 7(3): 381-92. doi: $10.1021 / \mathrm{ma} 60039 \mathrm{a} 022$.

40. Accelys Inc.. 2011. Amorphous Cell, Version 6.0.

41. Tundo, Pietro, and Maurizio Selva. 2002. "The Chemistry of Dimethyl Carbonate." Accounts of Chemical Research 35(9): 706-16. doi:10.1021/ar010076f.

42. Seward, Ralph P., and Ernest C. Vieira. 1958. "The Dielectric Constants of Ethylene Carbonate and of Solutions of Ethylene Carbonate in Water, Methanol, Benzene and 
Propylene Carbonate." The Journal of Physical Chemistry 62 (1): 127-28. doi:10.1021/j150559a041.

43. Vidulich, George A., Douglas Fennell Evans, and Robert L. Kay. 1967. "The Dielectric Constant of Water and Heavy Water between 0 and 40.degree." The Journal of Physical Chemistry 71(3): 656-62. doi:10.1021/j100862a028.

44. Sun, H. 1998. "COMPASS: An Ab Initio Force-Field Optimized for Condensed-Phase Applications: Overview with Details on Alkane and Benzene Compounds." The Journal of Physical Chemistry B 102(38): 7338-64. doi:10.1021/jp980939v.

45. Karasawa, Naoki, and William A. Goddard. 1989. "Acceleration of Convergence for Lattice Sums." The Journal of Physical Chemistry 93(21): 7320-27. doi:10.1021/j100358a012.

46. Wang, Junmei, Romain M. Wolf, James W. Caldwell, Peter A. Kollman, and David A. Case. 2004. "Development and Testing of a General Amber Force Field." Journal of Computational Chemistry 25 (9): 1157-74. doi:10.1002/jcc.20035.

47. Berendsen, H. J. C., J. R. Grigera, and T. P. Straatsma. 1987. "The Missing Term in Effective Pair Potentials." The Journal of Physical Chemistry 91 (24): 6269-71. doi:10.1021/j100308a038.

48. Wilkes, C. E., J. W. Summers, C. A. Daniels, and M. T. Berard. 2005. PVC Handbook. Hanser Verlag.

49. Theodorou, Doros N., and Ulrich W. Suter. 1985. "Detailed Molecular Structure of a Vinyl Polymer Glass." Macromolecules 18(7): 1467-78. doi:10.1021/ma00149a018.

50. Bernardin III, Frederick E., and Gregory C. Rutledge. 2007. "Simulation of Mechanical Properties of Oriented Glassy Polystyrene." Polymer 48(24): 7211-20. doi:10.1016/j.polymer.2007.09.034.

51. Lacks, Daniel J., and Gregory C. Rutledge. 1995. "Temperature Dependence of Structural and Mechanical Properties of Isotactic Polypropylene." Macromolecules 28(4): 1115-20. doi:10.1021/ma00108a045.

52. Karasawa, Naoki, Siddharth Dasgupta, and William A. Goddard. 1991. "Mechanical Properties and Force Field Parameters for Polyethylene Crystal." The Journal of Physical Chemistry 95(6): 2260-72. doi:10.1021/j100159a031.

53. Sorensen, R. A., W. B. Liau, L. Kesner, and R. H. Boyd. 1988. "Prediction of Polymer Crystal Structures and Properties: Polyethylene and Poly(oxymethylene)." Macromolecules 21(1) : 200-208. doi:10.1021/ma00179a039.

54. Tashiro, Kohji, Masamichi Kobayashi, and Hiroyuki Tadokoro. 1992. "Vibrational Spectra and Theoretical Three-Dimensional Elastic Constants of Isotactic Polypropylene Crystal: An Important Role of Anharmonic Vibrations." Polymer Journal 24 (9): 899-916. doi:10.1295/polymj.24.899.

55. Miyazawa, Tatsuo. 1964. "Theory of Normal Vibrations of Helical Polymers and Vibrational Assignment of the Infrared Spectra of Isotactic Polypropylene." Journal of Polymer Science Part C: Polymer Symposia 7 (1): 59-84. doi:10.1002/polc.5070070106. 
56. Tadokoro, Hiroyuki, Masamichi Kobayashi, Minoru Ukita, Katsutoshi Yasufuku, Shunsuke Murahashi, and Tatsuo Torii. 1965. "Normal Vibrations of the Polymer Molecules of Helical Conformation. V. Isotactic Polypropylene and Its Deuteroderivatives." The Journal of Chemical Physics 42 (4): 1432-49. doi:10.1063/1.1696134.

57. Sawatari, Chie, and Masaru Matsuo. 1989. "Temperature Dependence of Crystal Lattice Modulus and Dynamic Mechanical Properties of Ultradrawn Polypropylene Films." Macromolecules 22(7): 2968-73. doi:10.1021/ma00197a016.

58. Peguy, A., and R. S. Manley. 1984. "Ultra-Drawing of High Molecular-Weight Polypropylene." Polymer Communications 25(2):39-42.

59. Hsu, S. L., S. Krimm, S. Krause, and G. S. Y. Yeh. 1976. "Longitudinal Acoustic Mode in Isotactic Polypropylene.” Journal of Polymer Science: Polymer Letters Edition 14(4): 195200. doi:10.1002/pol.1976.130140403.

60. Buell, Sezen, Krystyn J. Van Vliet, and Gregory C. Rutledge. 2009. "Mechanical Properties of Glassy Polyethylene Nanofibers via Molecular Dynamics Simulations." Macromolecules 42(13): 4887-95. doi:10.1021/ma900250y.

61. Doruker, Pemra. 2002. "Simulation of Polyethylene Thin Films Composed of Various Chain Lengths." Polymer 43(2): 425-30. doi:10.1016/S0032-3861(01)00430-X.

62. Gautieri, Alfonso, Simone Vesentini, Alberto Redaelli, and Markus J. Buehler. 2011. "Hierarchical Structure and Nanomechanics of Collagen Microfibrils from the Atomistic Scale Up." Nano Letters 11(2): 757-66. doi:10.1021/nl103943u.

63. Sen, Fatih G., Yue Qi, Adri C. T. van Duin, and Ahmet T. Alpas. 2013. "Oxidation Induced Softening in Al Nanowires." Applied Physics Letters 102(5): 051912. doi:10.1063/1.4790181.

64. Wittkop, M., S. Kreitmeier, and D. Göuritz. 1995. "On the Deformation of Hard Elastic Polymers - Applicability of the Coil-Strand-Transition Model.” Acta Polymerica 46(4): 319-27. doi:10.1002/actp.1995.010460406.

65. Peabody, Christina, and Craig B. Arnold. 2011. "The Role of Mechanically Induced Separator Creep in Lithium-Ion Battery Capacity Fade.” Journal of Power Sources 196 (19): 8147-53. doi:10.1016/j.jpowsour.2011.05.023. 
Table 1. The top view and side view of fully relaxed atomic structures of crystalline, crystalline nanofiber, oriented amorphous infinitely long chain nanofiber, and oriented amorphous finite chain nanofiber. Each chain was highlighted with different color. Vacuum layer was added in the simulation cell along $\mathrm{x}$ and $\mathrm{y}$ direction to simulate isolated nanofibers, although the simulation cell boundaries are not shown in the top views. The length of the scale bar is $5 \mathrm{~nm}$. 


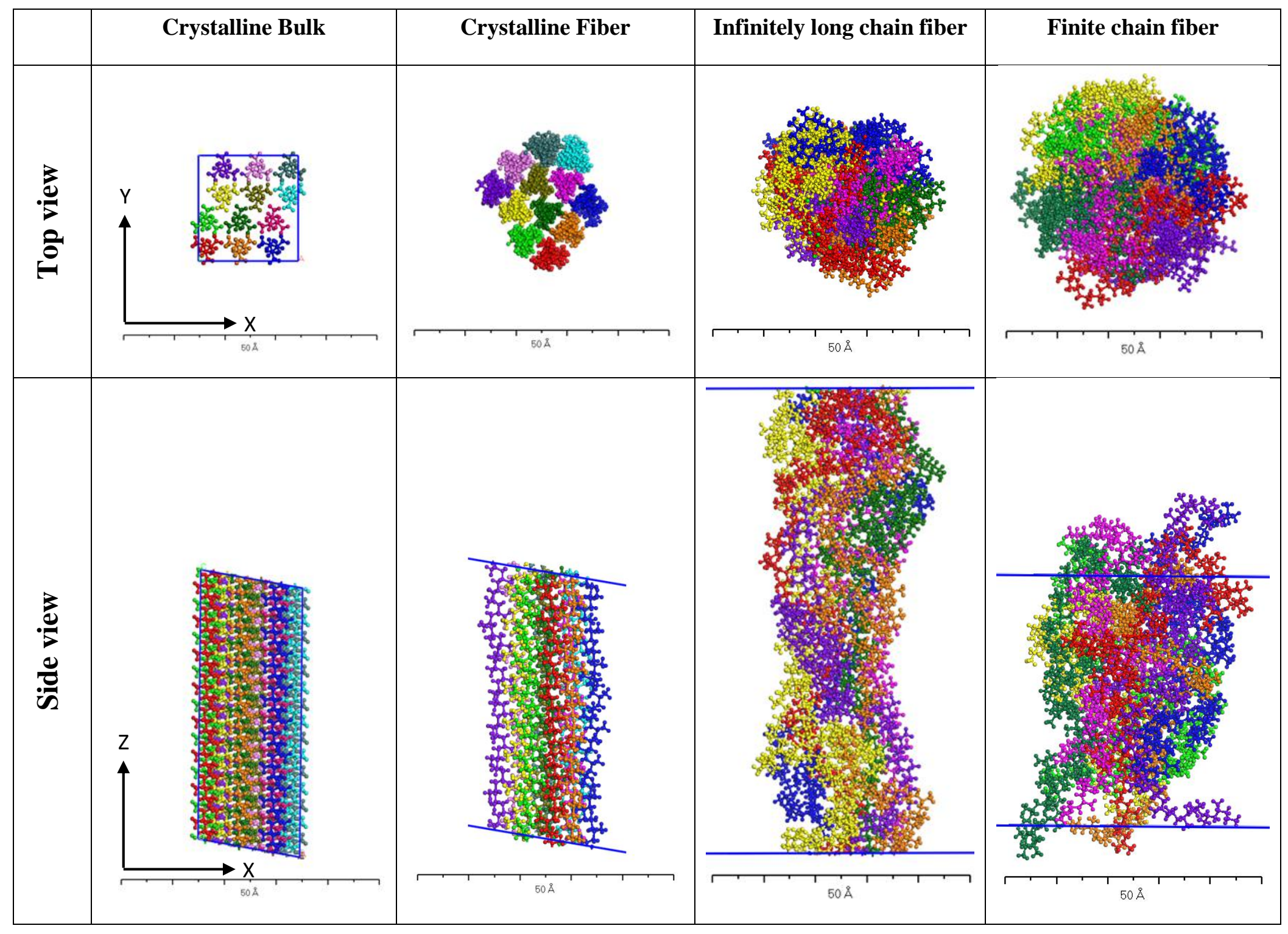


Table 2. Simulation size

\begin{tabular}{c|cccc}
\hline \hline Simulation size & Structure & In vacuum & In DMC & In water \\
\hline $\begin{array}{c}\text { Simulation cell } \\
\text { size } \mathrm{X} \times \mathrm{Y} \times \mathrm{Z} \\
(\AA)\end{array}$ & Crystalline bulk & $19.98 \times 20.78 \times 51.96$ & & \\
& Crystalline fiber & $39.96 \times 41.56 \times 51.96$ & $39.96 \times 41.56 \times 51.96$ & \\
& Infinitely long chain fiber & $80 \times 80 \times 90.34$ & $55 \times 55 \times 90.34$ & \\
& Finite chain fiber & $57.72 \times 57.72 \times 48.47$ & $63.86 \times 63.86 \times 48.47$ & $63.86 \times 63.86 \times 48.47$ \\
\hline $\begin{array}{c}\text { Total number of } \\
\text { atoms }\end{array}$ & Crystalline bulk & 2592 & & \\
& Crystalline fiber & 2592 & 7968 & \\
& Infinitely long chain fiber & 5832 & 23364 & 19501 \\
\hline $\begin{array}{c}\text { Number of } \\
\text { solvent } \\
\text { molecule } \\
\text { packed }\end{array}$ & Finite chain fiber & 5776 & 448 & \\
\hline
\end{tabular}


Table 3. Simulation time required for convergence and used at each strain step to calculate the stress-strain for nanofibers in different environments.

\begin{tabular}{|c|c|c|c|c|}
\hline Time & Structure & In vacuum & In DMC & In water \\
\hline \multirow{4}{*}{$\begin{array}{c}\text { Simulation time at } \\
\text { zero strain }(\mathrm{ps})\end{array}$} & Crystalline bulk & 50 & & \multirow{4}{*}{3000} \\
\hline & Crystalline fiber & 180 & 120 & \\
\hline & Infinitely long chain fiber & 300 & 600 & \\
\hline & Finite chain fiber & 50 & 3000 & \\
\hline \multirow{4}{*}{$\begin{array}{l}\text { Time starts to } \\
\text { converge at zero } \\
\text { strain (ps) }\end{array}$} & Crystalline bulk & 30 & & \\
\hline & Crystalline fiber & 160 & 100 & \multirow{3}{*}{2000} \\
\hline & Infinitely long chain fiber & 200 & 410 & \\
\hline & Finite chain fiber & 30 & 1500 & \\
\hline \multirow{4}{*}{$\begin{array}{l}\text { Simulation time } \\
\text { at each } 1 \% \text { strain } \\
\text { increment (ps) }\end{array}$} & Crystalline bulk & 30 & & \\
\hline & Crystalline fiber & 40 & 40 & \multirow{3}{*}{500} \\
\hline & Infinitely long chain fiber & 300 & 600 & \\
\hline & Finite chain fiber & 50 & 400 & \\
\hline \multirow{4}{*}{$\begin{array}{c}\text { Time starts to } \\
\text { converge at each } \\
1 \% \text { strain } \\
\text { increment }(\mathrm{ps})\end{array}$} & Crystalline bulk & 20 & & \multirow{4}{*}{350} \\
\hline & Crystalline fiber & 30 & 30 & \\
\hline & Infinitely long chain fiber & 200 & 500 & \\
\hline & Finite chain fiber & 30 & 250 & \\
\hline \multirow{4}{*}{$\begin{array}{l}\text { Resulted strain } \\
\operatorname{rate}\left(\mathrm{s}^{-1}\right)\end{array}$} & Crystalline bulk & $3.3 \times 10^{8}$ & & \\
\hline & Crystalline fiber & $2.5 \times 10^{8}$ & $2.5 \times 10^{8}$ & \\
\hline & Infinitely long chain fiber & $3.33 \times 10^{7}$ & $1.67 \times 10^{7}$ & \\
\hline & Finite chain fiber & $2 \times 10^{8}$ & $2.5 \times 10^{7}$ & $2 \times 10^{7}$ \\
\hline
\end{tabular}


Table 4. Young's modulus calculations for bulk crystal, crystalline fiber, infinitely long chain fiber, finite chain fiber in vacuum, in DMC and in water. (m) means modeling results and (e) means experimental results.

\begin{tabular}{|c|c|c|c|c|}
\hline \multirow{2}{*}{ Structure } & \multicolumn{2}{|r|}{ In Vacuum } & \multirow{2}{*}{$\begin{array}{c}\text { In DMC } \\
\text { Our } \\
\operatorname{model}(\mathrm{GPa})\end{array}$} & \multirow{2}{*}{$\begin{array}{c}\text { In Water } \\
\text { Our } \\
\text { model }(\mathrm{GPa})\end{array}$} \\
\hline & $\begin{array}{c}\text { Our } \\
\operatorname{model}(\mathrm{GPa})\end{array}$ & $\begin{array}{l}\text { Experiment (e)/Previous } \\
\text { molecular modeling (m) }\end{array}$ & & \\
\hline $\begin{array}{l}\text { Bulk crystal } \\
\qquad E_{X}\end{array}$ & 3.81 & $7.14\left(\mathrm{~m}^{\mathrm{a}}\right) ; 8.19\left(\mathrm{~m}^{\mathrm{b}}\right) ; 7.13\left(\mathrm{~m}^{\mathrm{c}}\right)$ & & \\
\hline $\mathrm{E}_{\mathrm{Y}}$ & 3.47 & $7.14\left(\mathrm{~m}^{\mathrm{a}}\right) ; 9.52\left(\mathrm{~m}^{\mathrm{b}}\right) ; 5.35\left(\mathrm{~m}^{\mathrm{c}}\right)$ & & \\
\hline $\mathrm{E}_{Z}$ & 49.5 & $36 \sim 42\left(\mathrm{e}^{\mathrm{d}}\right)$ & & \\
\hline Crystalline fiber & 43.4 & --- & 46.5 & \\
\hline $\begin{array}{l}\text { Infinitely long chain } \\
\text { fiber }\end{array}$ & 0.66 & --- & 0.07 & \\
\hline Finite chain fiber & 0.29 & --- & $0.01^{\mathrm{f}}$ & 0.59 \\
\hline
\end{tabular}

${ }^{\mathrm{a}}[51,52] \cdot{ }^{\mathrm{b}}[51,53] \cdot{ }^{\mathrm{c}}[54-56] \cdot{ }^{\mathrm{d}}[57-59]$. 
Table 5. Relaxed structure for crystalline fiber, infinitely long chain fiber and finite chain fiber in vacuum, in DMC and in water at zero strain. The structure of finite chain fiber was from $3 \mathrm{~ns}$ relaxation result. The fibers were colored in Green. The fibers and solvent structures are shown separately when the fibers were submersed in solvent. The length of the scale bar is $5 \mathrm{~nm}$.

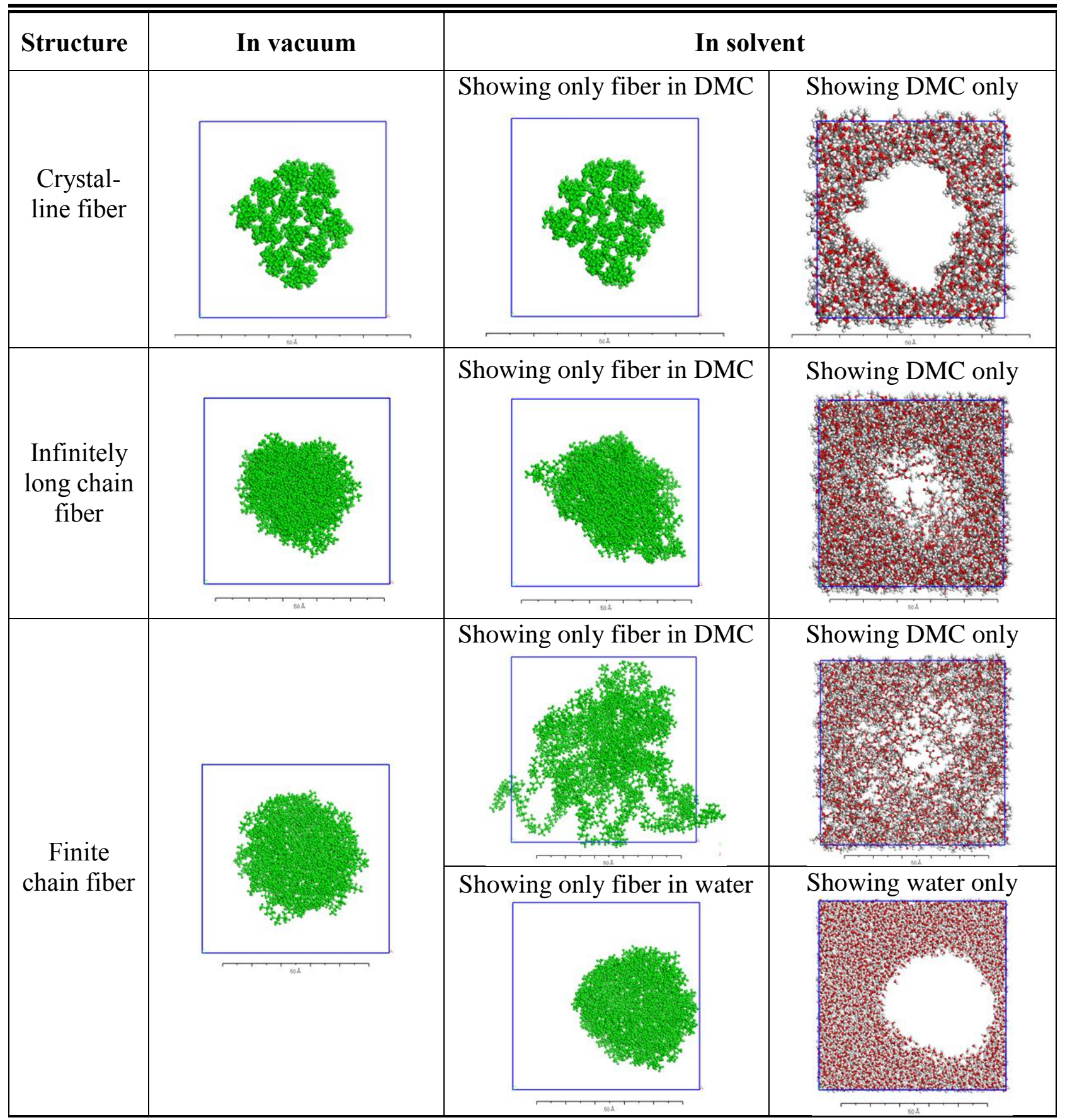




\section{Figures and Captions}

Figure 1. SEM image of Celgard 2400 imaged with a dual column focused ion beam (FIB)-SEM (Carl Zeiss Auriga CrossBeam)

Figure 2. Illustration of packing solvent molecules into the vacuum around infinitely long chain fiber. (a) shows that a solvent accessible surface was created to wrap the fiber and (b) shows 1461 DMC molecules were packed into the space outside the accessible surface.

Figure 3. Simulated true stress-strain curves for the (a) crystalline fiber, (b) oriented amorphous infinitely long chain fiber, and (c) oriented amorphous finite chain fiber in vacuum, DMC and water. Young's modulus E was obtained by linear fitting of the stress-strain curve.

Figure 4. Local density profiles of (a) crystalline fiber, (b) oriented amorphous infinitely long chain fiber, and (c) oriented amorphous finite chain fiber in vacuum, DMC and water. 


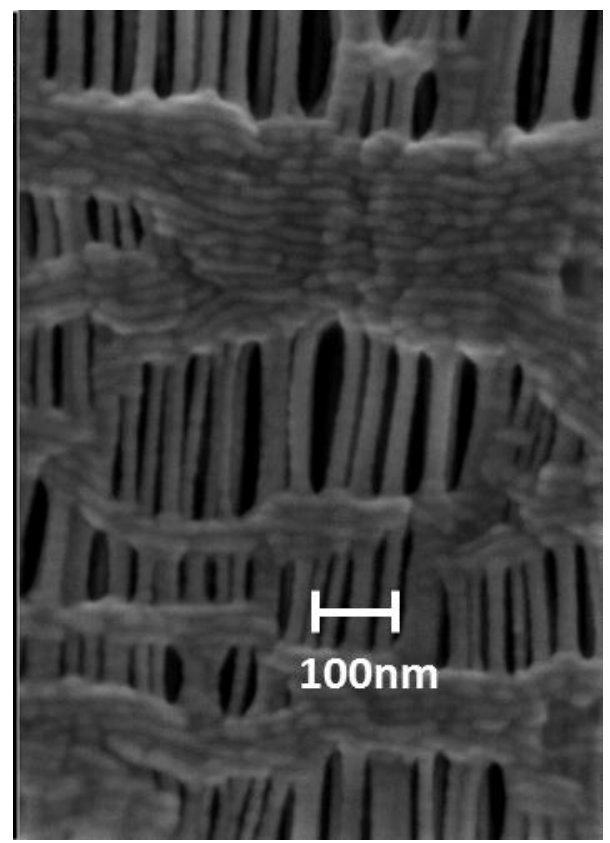

Figure 1. SEM image of Celgard 2400 imaged with a dual column focused ion beam (FIB)-SEM (Carl Zeiss Auriga CrossBeam) 


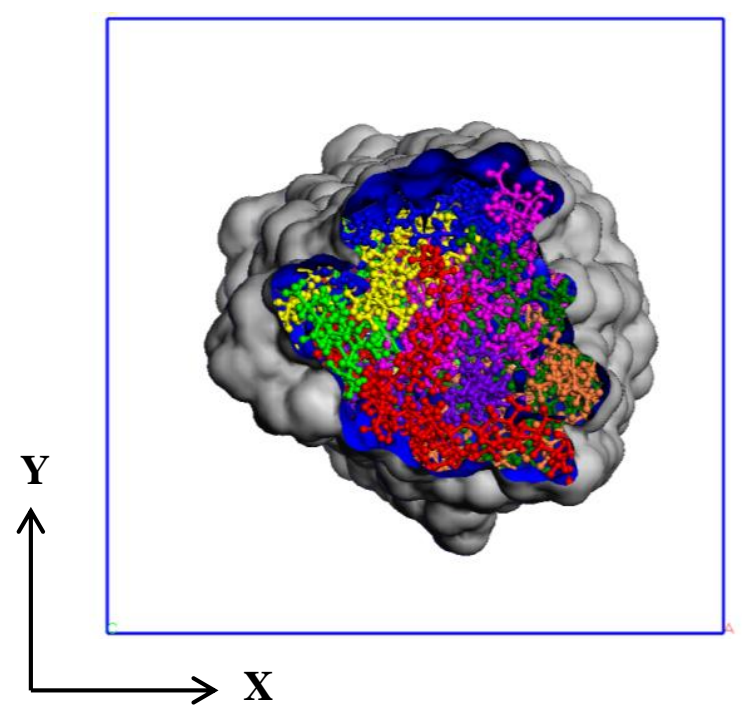

(a) Creating solvent accessible surface

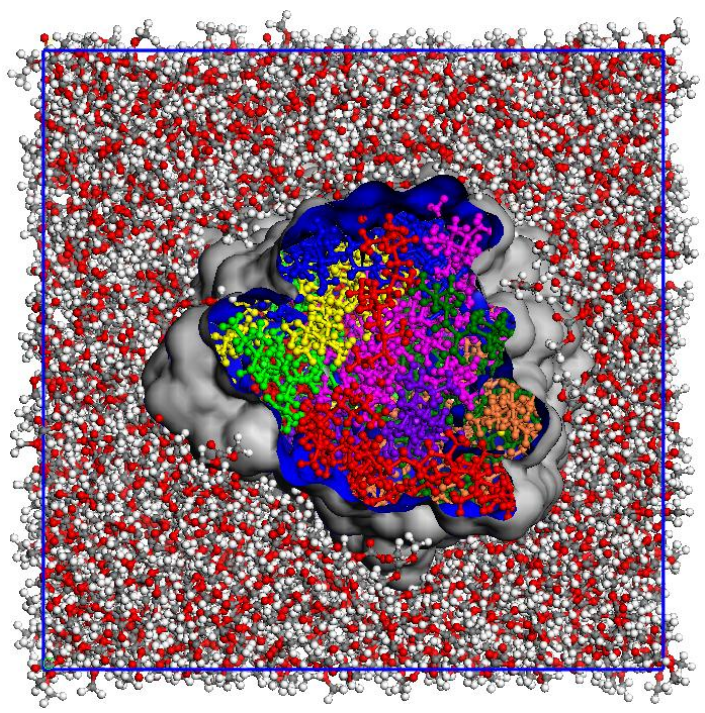

(b) Packing liquid molecules

Figure 2. Illustration of packing solvent molecules into the vacuum around infinitely long chain fiber. (a) shows that a solvent accessible surface was created to wrap the fiber and (b) shows 1461 DMC molecules were packed into the space outside the accessible surface. 


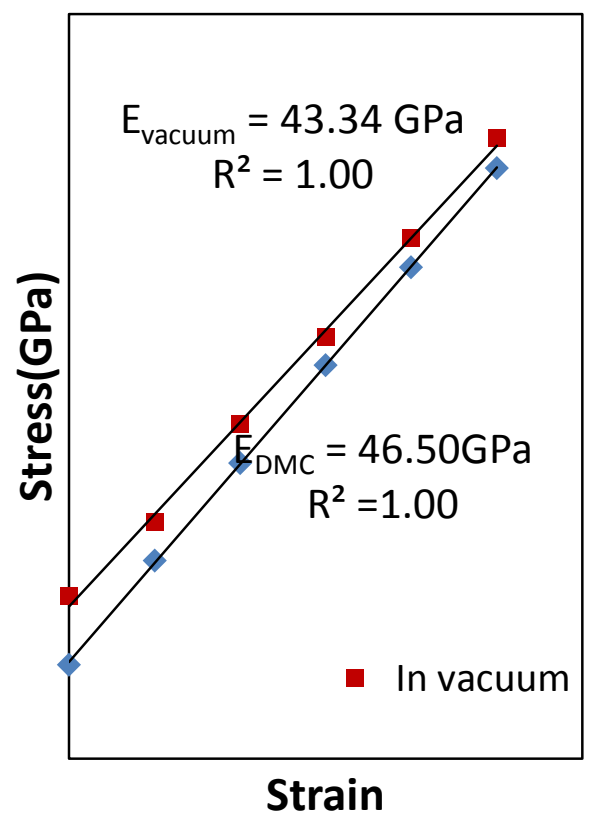

(a)

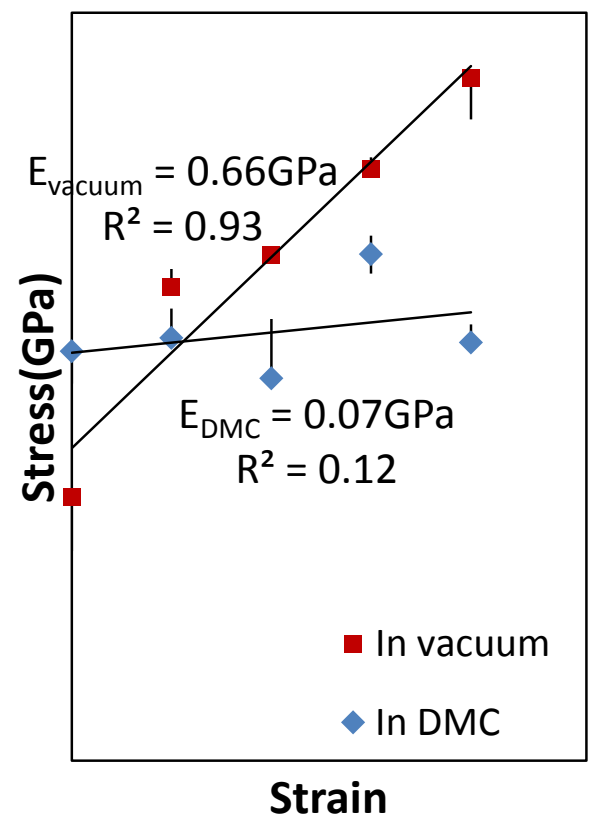

(b) 


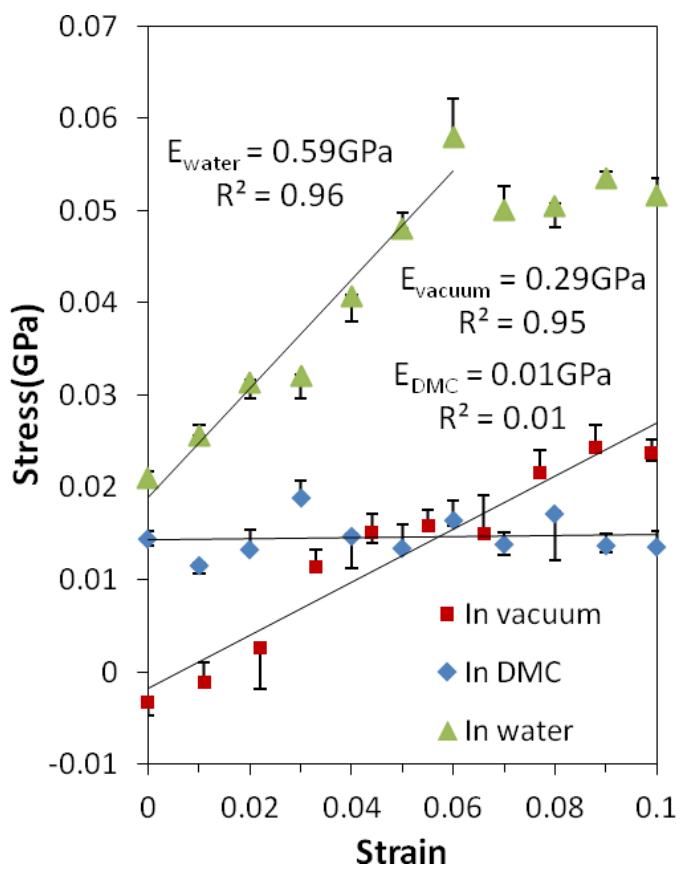

(c)

Strain

Figure 3. Simulated true stress-strain curves for the (a) crystalline fiber, (b) oriented amorphous infinitely long chain fiber, and (c) oriented amorphous finite chain fiber in vacuum, DMC and water. Young's modulus E was obtained by linear fitting of the stress-strain curve. 
(a) Density profile of crystalline fiber

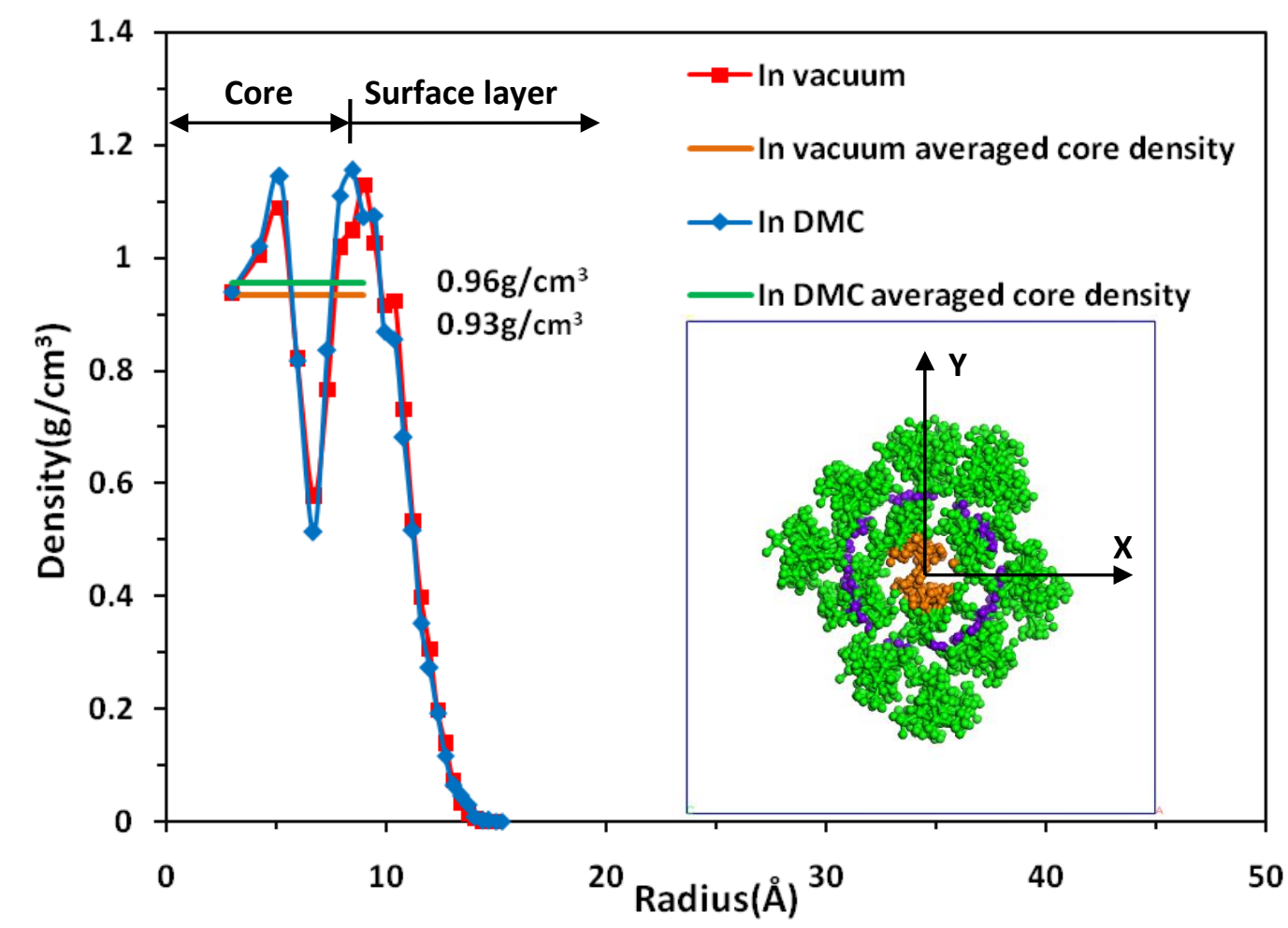

(b) Density profile of infinitely long chain fiber

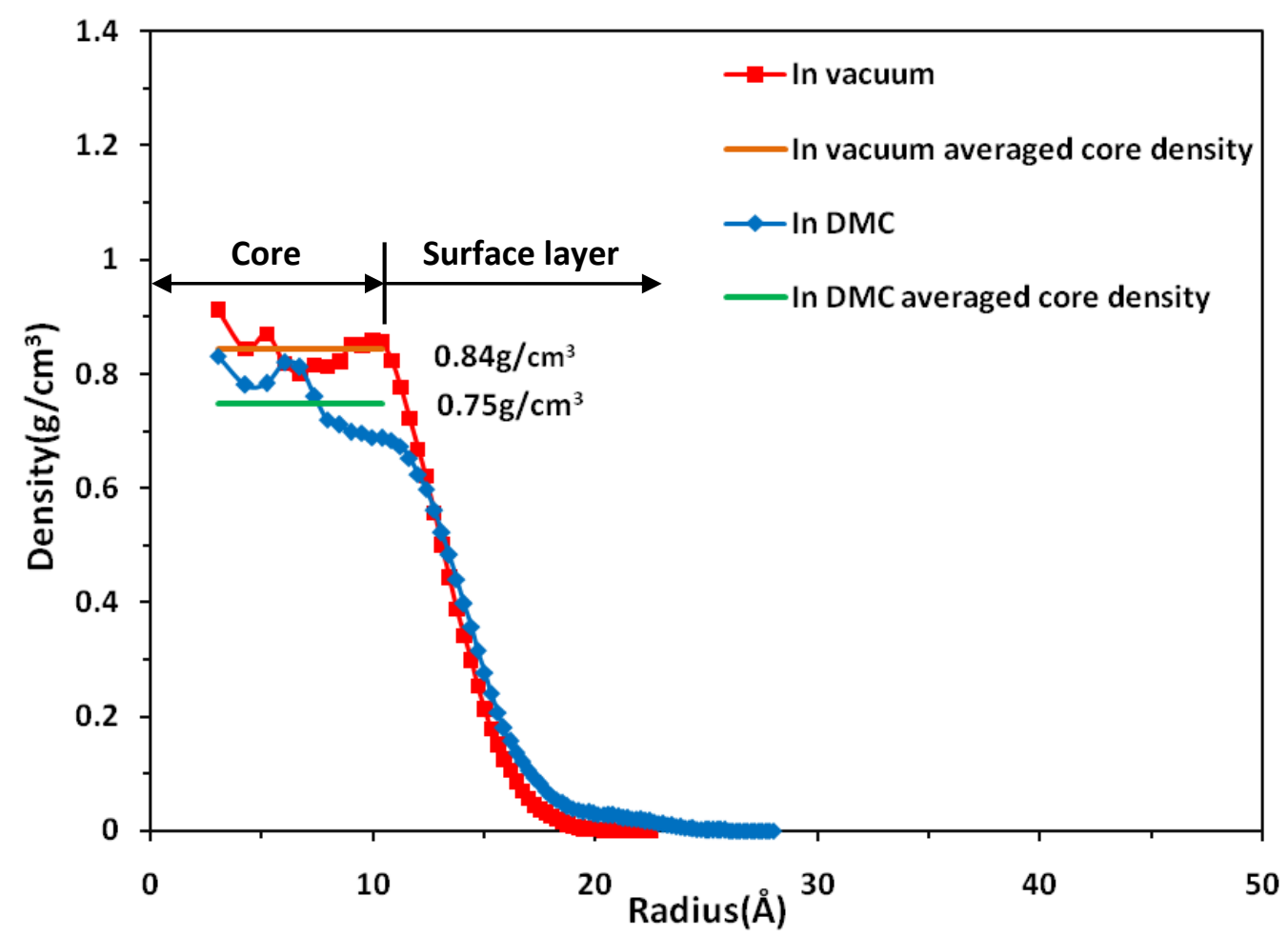


(c) Density profile of finite chain fiber

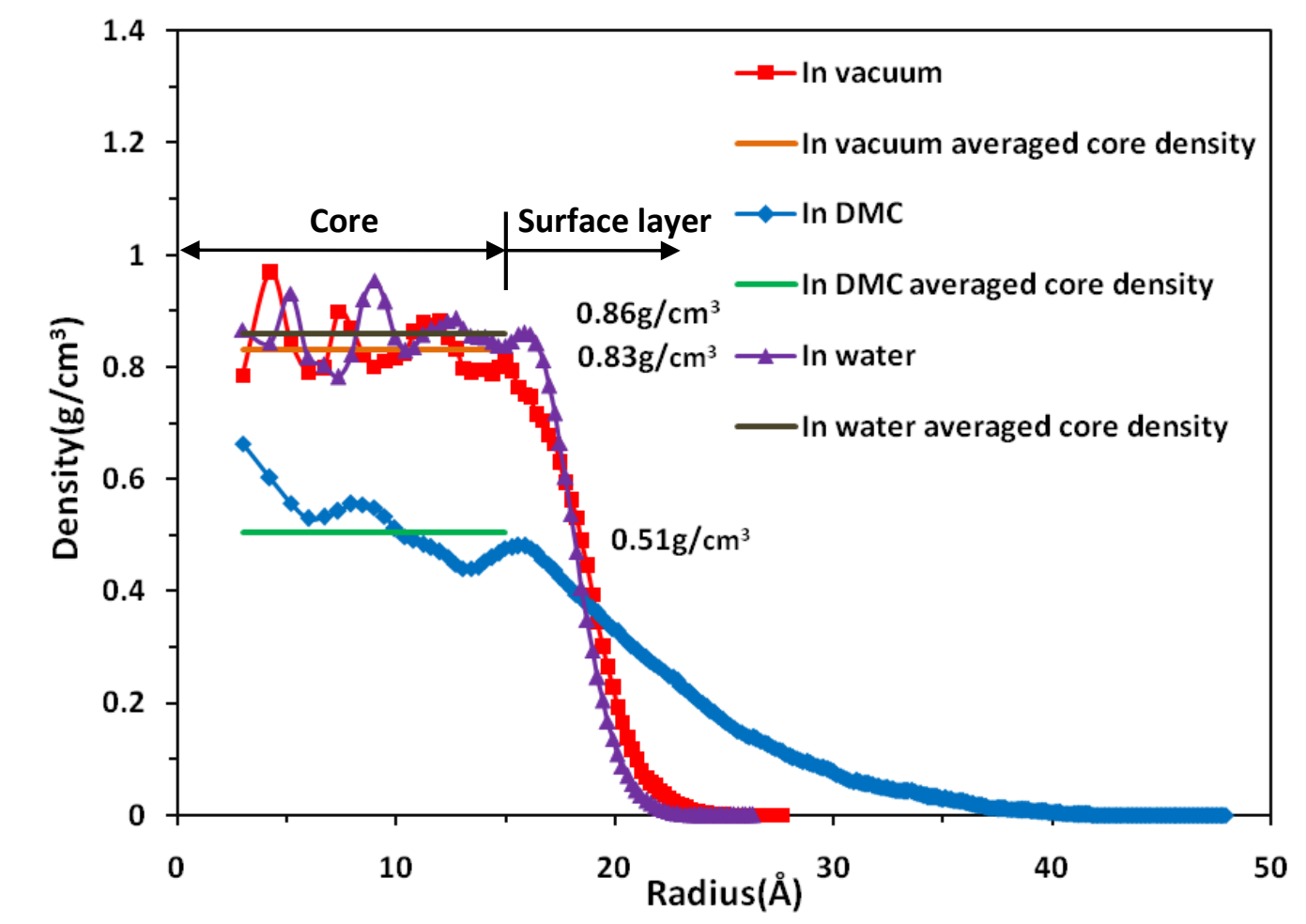

Figure 4. Local density profiles of (a) crystalline fiber, (b) oriented amorphous infinitely long chain fiber, and (c) oriented amorphous finite chain fiber in vacuum, DMC and water. 